\title{
Basiliximab induction therapy in kidney transplantation: Benefits for long term allograft function after 10 years?
}

\author{
Martina Koch \\ Thomas Becker \\ Rainer Lueck \\ Michael Neipp \\ Juergen Klempnauer \\ Bjoern Nashan \\ Klinik fuer Allgemein-, Viszeral- und \\ Transplantationschirurgie, \\ Medizinische Hochschule Hannover, \\ Hannover, Germany
}

\begin{abstract}
The interleukin-2 receptor antagonist basiliximab has proven in large clinical trials to be safe and effective to reduce acute rejections in the first year after renal transplantation. Since acute rejections are a risk factor for chronic graft loss, their effective reduction might have a positive effect on long term allograft survival. So far data is spares to prove this hypothesis and 10-year follow up on basiliximab induction therapy is not available. In our center, 41 patients were enrolled in the multicenter trial CHIB201 in 1995/96 comparing basiliximab vs no induction therapy after renal transplantation. We retrospectively analyzed the outcome of these patients after 10 years. The main reason for patient death with functioning graft were infectious complications (basiliximab: 3/20, placebo 1/19), 21\% of all patients developed cancer without an obvious correlation to specific immunosuppression. Death censored 10-year graft survival was equivalent in both groups: $65 \%$ in the basiliximab and $68 \%$ in the placebo group with a mean s-creatinine-clearance of 60 and $44 \mathrm{ml} / \mathrm{min}$. In this small study patient and graft survival was equivalent 10 years after transplantation comparing basiliximab induction therapy and placebo.
\end{abstract}

Keywords: basiliximab, induction therapy, long term graft survival, renal transplantation

\section{Introduction}

Basiliximab (Simulect ${ }^{\circledR}$ ) is a chimeric monoclonal antibody directed against the alpha chain of the interleukin-2 receptor (IL-2R) and competitively inhibits IL-2 dependent T-cell activation in acute allograft rejection. ${ }^{1}$ Basiliximab induction therapy has proven in large clinical trials to be effective to reduce the rate of acute rejections and to allow sparing of calcinurin inhibitors (CNI) and steroides in the early postoperative period after renal transplantation (RTx) with similar tolerability to placebo..$^{2-4}$ Most studies focus on acute rejection rates and one year allograft survival, but less is known about the impact of induction therapy with IL-2R antagonists on long term allograft survival.

Since it is known that acute rejections are a risk factor for chronic transplant dysfunction, ${ }^{5-7}$ an effective induction therapy might have a beneficial long-term effect on renal function.

Other than induction therapies with antithymocyte globulin (ATG) or anti-CD3 monoclonal antibody (OKT3) ${ }^{8,9}$ no significant increase in malignancies or infections was related with the application of basiliximab in the first years after RTx, ${ }^{4,10}$ but long term evaluations are sparse.

In this study we address the question if antibody induction therapy with basiliximab compared to no induction therapy leads to better long term renal allograft function and/or patient survival after 10 years. We retrospectively analyzed patients enrolled in a multicenter phase III trial (CHIB 201) ${ }^{3}$ at Hanover Medical School, Germany in 
1995/1996. Patients were treated either with basiliximab or placebo in combination with cyclosporine A (CyA) and steroides after first cadaveric renal transplantation and were followed over a 10-year period. The results of this study were compared with published long-term data available for other IL-2R antagonists.

\section{Methods}

\section{Study design and patients demographics}

We retrospectively analyzed the outcome of 41 recipients of primary cadaveric renal allografts enrolled in the CHIB 201 study at Hanover Medical School, Germany in 1995/1996. The primary objective of the CHIB 201 study was to analyze the rate of acute rejections in the first six months after kidney transplantation in combination with standard immunosuppressive therapy consisting of $\mathrm{CyA}$ and corticosteroids.

Twenty one patients received $20 \mathrm{mg}$ of basiliximab on days 0 and 4 post-transplantation and 20 patients received placebo. Induction therapy was combined with standard immunosuppressive therapy consisting of CyA and corticosteroids. There were no relevant differences between the placebo and the basiliximab group regarding demographic data and medical condition at time of transplantation. Gender, age, and reason for transplantation are shown in Table 1.

\section{Immunosuppression}

Initial immunosuppression consists of low doses of CyA and corticosteroids with or without basiliximab induction therapy. The mean CyA trough levels have been 130-150 ng/ml in both groups within the first three months in our center. In case of acute rejection treatment was performed with bolus injections of $500 \mathrm{mg}$ methylprednisolone given intravenously for three days without a change in baseline immunosuppression. If this was without success patients received $\mathrm{OKT} 3$ in a dose of $5 \mathrm{mg} /$ day for $7-8$ days. No routine biopsies were performed.

\section{Long term evaluation}

Patients were followed for 10 years by using clinical examination and laboratory tests done in our outpatient facility at least once a year. If visits in our centre were not possible records provided by the patients local nephrologists were used for long-term follow up.

Follow up was terminated at the time patients returned to dialysis or died.

Creatinine clearances were calculated using the Cockcroft-Gault formula: ${ }^{11}(140-$ age $) \times($ weight $/(72 \times$ creatinine $(\mu \mathrm{mol} / 1) / 88.4))$ for male and multiplied with 0.85 for female patients.

\section{Statistics}

Statistical significance of differences in renal function was calculated using Students t-test (Microsoft Excel, Microsoft Corp., Redmond, WA) and patient and graft survival was analyzed by Kaplan-Meier estimates using Wilcoxon signedrank tests. $\mathrm{P}<0.05$ was given as significant.

\section{Results}

\section{Patient and graft survival after 10 years}

As in the multicenter trial ${ }^{3}$ in the subgroup of patients analyzed here acute rejections in the first six month posttransplantation were significant higher in the placebo group ( $55 \%$ vs 29\%). No steroid resistant rejections occurred in the basiliximab group, while in the placebo group five patients were treated with OKT3.

In both groups one patient was lost for follow up within the first year after transplantation and was excluded from the long term analysis.

In the basiliximab group three patients $(3 / 20)$ died with functioning graft within 10 years after transplantation resulting

Table I Gender, age and reason for transplantation in the placebo and the basiliximab group

\begin{tabular}{lll}
\hline & Basiliximab group & Placebo group \\
\hline Male/female & $10 / 10$ & $13 / 6$ \\
Age at time of transplantation (years) & $46 \pm 12$ & $47 \pm 14$ \\
Reasons for transplantation: & & 5 \\
- Glomerulonephritis & 4 & 1 \\
- IgA nephritis & 4 & 4 \\
- Cystic kidney degeneration & 2 & 4 \\
Interstitial nephritis/pyelonephritis & 4 & 5 \\
Others/unknown & 6 & \\
\hline
\end{tabular}


in a patient survival of $85 \%$. Reasons for death were: sarcoma (three years post-RTx, age 68), Pneumococcus pneumonia (four years post-RTx, age 59) and sepsis (Pneumocystis carinii pneumonia, seven years post-transplantation in the age of 44 years).

Seven patients lost their grafts in the basiliximab group, due to the following reasons: two vascular complications immediately after transplantation, five patients returned to dialysis due to long term renal failure. Reasons were (biopsy confirmed): in three patients recurrent renal disease $(2 \times \operatorname{IgA}$ nephropathy, $1 \times$ light chain amyloidosis) five, six and seven years after transplantation and chronic rejection and/or CNI-toxicity in two patients after 10 years. 10/20 patients (50\%) had a functioning graft after 10 years (Figure 1). Death-censored graft survival in the basiliximab group was $65 \%$ after 10 years.

In the placebo group, patient survival was 95\% after 10 years; one $(1 / 19)$ patient died from sepsis three years after transplantation with functioning graft with 52 years. In the placebo group six graft losses occurred: One due to anemic infarction 20 days after transplantation, three patients lost their graft due to chronic rejection $(5,6$, and 9 years after RTx) and one patient due to hypertensive damage and CNI toxicity after 6 years. Considering one patient dying with functioning graft $12 / 19$ patients (63\%) had a functioning graft 10 years after transplantation (Figure 1). Death-censored graft survival in the placebo group was $68 \%$ after 10 years.

For both groups, 22 of 39 kidneys were functioning 10 years after transplantation, this is a graft survival of $56 \%$ for all patients, death-censored $67 \%$.

\section{Renal function after 10 years}

In the basiliximab group, 10 patients with a functioning graft have S-creatinine values between 93 and $158 \mu \mathrm{mol} / 1$ and a mean creatinine-clearance of $60 \mathrm{ml} / \mathrm{min}$. In six patients renal function was improved or stable (clearance $\pm 10 \mathrm{ml} / \mathrm{min}$ ) from year 1 to year 10 after transplantation, in four patients renal function was decreasing (Figure 2a).

In the placebo group 12 patients with functioning grafts had s-creatinine values between 78 and $332 \mu \mathrm{mol} / 1$ and a mean clearance of $44 \mathrm{ml} / \mathrm{min}$. Six grafts displayed an improved or stable function (clearance $\pm 10 \mathrm{ml} / \mathrm{min}$ compared to year one) over ten years and in six patients renal function declined (Figure 2b).

\section{Immunosuppression}

In the basiliximab group, no OKT3 treatment was needed for acute steroid-resistant rejection, while five patients in the placebo group received OKT3.

Ten years after transplantation, 9/10 patients with functioning graft in the basiliximab group were on CyA-based immunosuppression, five of them in combination with either mycophenolate mofetil (MMF) or azathioprine (AZA). One patient takes rapamycine due to the diagnosis of melanoma.

In the placebo group 9/12 patients are treated with CyA-based immunosuppression, three of them in combination with MMF or AZA. Two patients are on tacrolimus-based immunosuppression and one patient with kaposi-sarcoma takes AZA and steroids only.

Four of five placebo-patients treated with OKT3 had functioning grafts after 10 years.

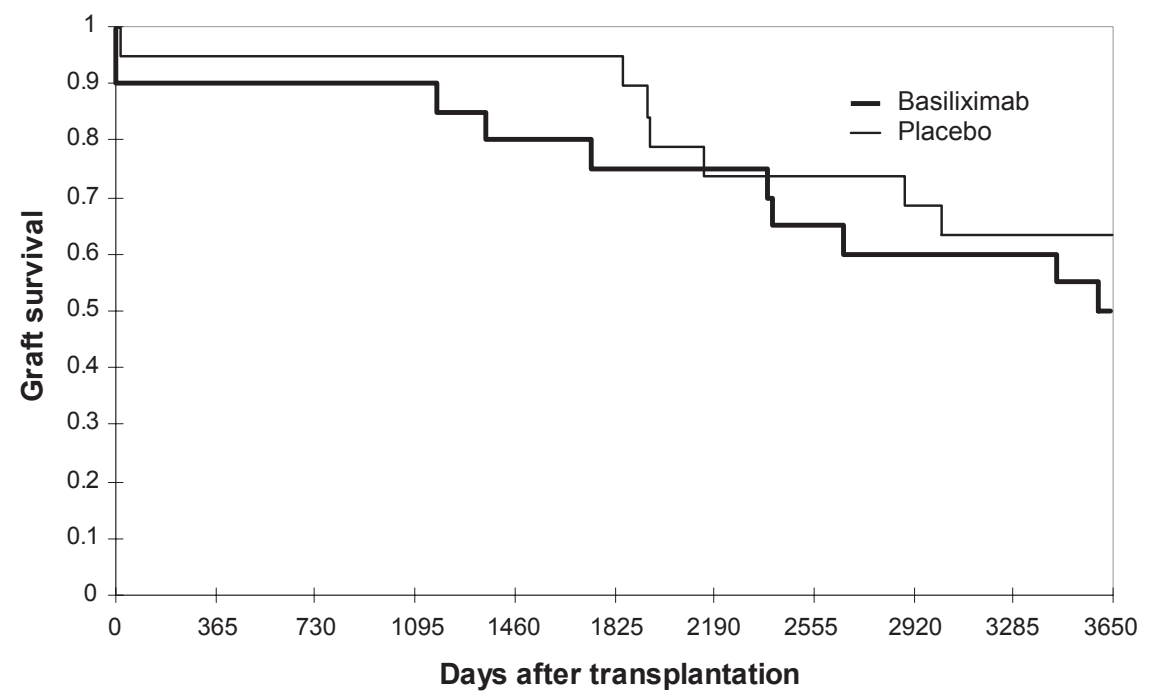

Figure I Ten-year graft survival in the basiliximab and the placebo group. There was no significant difference in graft survival between both groups. 

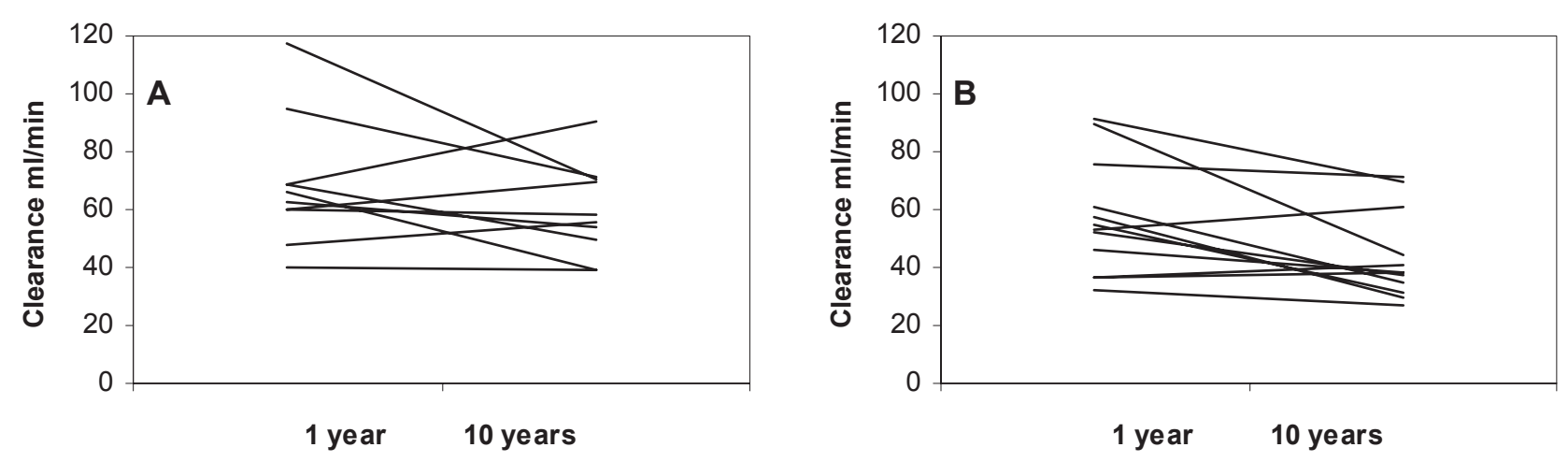

Figure 2 Changes in creatinine-clearance from year one to year ten after transplantation in patients with functioning graft at the end of the observation period. A) basiliximab group, B) placebo group.

\section{Malignancies}

In the basiliximab group, six malignancies were reported in five patients: One patient died from sarcoma three years post-RTx, a second was nephrectomized due to renal cell carcinoma in his own kidney four years post-transplantation, a third patient developed spinocellular carcinoma of the upper lip after eight years, a forth patient developed a T1 melanoma and a basalioma after nine years and a female patient was diagnosed with lung and liver metastasis of a carcinoma of the vagina after 10 years.

In the placebo group, three malignancies occurred. One patient developed kaposi sarcoma 18 months post-transplantation, and is now doing well 10 years post-RTx, a second patient developed spinocellular carcinoma of the lower lip after five years, and a third patient suffered from bladder carcinoma in the eighth year after transplantation.

In both groups, three nonmelanoma skin cancers and six other cancers including melanoma were found in $8 / 39(21 \%)$ patients. Seven of eight patients are alive with functioning grafts after 10 years.

\section{Discussion}

The IL-2 receptor antagonist (IL-2RA) basiliximab has proven to be effective in reducing acute rejection episodes in large double blind multi center trials without significant side effects. ${ }^{2,3}$ Due to these promising results, basiliximab is now commonly used, but sparse analysis on its long-term effects exist.

A report on the murine IL-2RA BT563 vs placebo after renal transplantation shows a comparable 10 -year patient and graft survival without increase in malignancies ${ }^{12}$ and a study comparing induction therapy with the rat IL-2R antagonist Lo-tact-1 and ATG demonstrated no difference in patient and graft survival after 10 years, but a tendency to less chronic rejection in the Lo-tact group..$^{13}$
No 10-year follow-up data on basiliximab is available at the moment. A five year analysis by Kyllönen and colleagues compares basiliximab-induction with ATG-induction and standard immunosuppression without induction primarily to analyze effects on delayed graft function, showing no difference between both induction groups for patient and graft survival, but a superior five year graft survival for the basiliximab group compared to induction-free immunosuppression. ${ }^{14}$

Taken together, the results of these studies do not clearly demonstrate a long-term benefit for IL-2R antagonists compared to other induction therapies or to no induction regarding patient or graft survival.

The CHIB 201 study, we used for retrospective analysis, was designed to compare basiliximab induction versus no induction therapy in combination with a low dose dual basic immunosuppression consisting only of CyA and corticosteroids. The study demonstrated an excellent reduction of acute rejections for all patients ${ }^{3}$ and the patients included in this sub-analysis despite of relatively low CyA levels in our centre. The number of patients requiring urbason bolus therapy for acute rejection within six months was reduced in the basiliximab group and more important no OKT3 or other antibody therapy was needed in the basiliximab group for treatment of steroid resistant rejection.

With regard to the common suggestion of acute rejections as a risk factor for chronic graft dysfunction, our hope was to improve long term allograft survival in patients free of acute rejections, but as in the studies mentioned above, we found comparable patient survival of $85 \% / 95 \%$ and deathcensored graft survival of $65 \% / 68 \%$ in the basiliximab and placebo group. Neither patient nor graft survival was significantly different. This result may underline the fact that acute rejections are only one risk factor in the multifactorial process of chronic graft loss. 
By taking a closer look at each patient, it is remarkable that in patients of the placebo group diagnosis of early acute rejection seems not to be necessarily correlated with worse long term graft function. All four patients after successful OKT3 treatment due to steroid-resistant rejection have functioning grafts after 10 years.

Kidney function in patients receiving basiliximab tended to be better after 10 years, but in both groups five chronic grafts losses occurred. They comprise of recurrence of primary disease as well as chronic rejection and/or CNI toxicity in both groups, again showing the multifactorial ethiology of chronic allograft dysfunction.

Other than in registry data, main reasons for patients' death in this study were not cardiovascular events, but infectious complications years after transplantation in both groups.

Malignancies become a challenge after transplantation since time of immunosuppression increases due to improved patient survival, as well as rising numbers of retransplanted and young patients. The incidence of most types of cancers is increased after transplantation and the number of malignancies found in our study is in accordance with reported rats of non melanoma skin and other cancers. ${ }^{15-17}$ An association of malignancies and immunosuppression in general is clearly proven, but a correlation with defined drugs is more difficult. In this study the sarcoma was found in a patient on tacrolimus based triple immunosuppression, while the other patients with malignomas in the basiliximab group were on dual therapy. One patient after OKT3 treatment in the placebo group developed cancer (urothel carcinoma). The patient diagnosed with a kaposi sarcoma was a placebo patient, who received an acute rejection treatment with steroids and was switched to tacrolimus based immunosuppression for the same reason within the first month post-RTx. After 10 years of immunosuppression, at least $1 / 3$ of patients suffered from cancer including skin cancers, but luckily only one patient died.

In summary we achieved a comparable patient and graft survival 10 years after renal transplantation with and without basiliximab induction therapy. While graft function in the basiliximab group tended to be better, the number of graft losses due to chronic damage including recurrence of primary disease was equal in both groups. Our results are consistent with reports available for other IL-2R antagonists and are not able to prove an improved long term graft or patient survival after basiliximab induction therapy. Since these are the only 10-year results available comparing basiliximab induction therapy with placebo and the number of patients analyzed is small, the conclusions from this study must be drawn carefully. Although basiliximab induction therapy is commonly used today, it seems worth to rethink the indication of induction therapies and to focus on further investigation on the effects of different immunosuppression on long term allograft survival.

\section{Acknowledgments}

MK collected and analyzed data and wrote the paper. TB, RL, $\mathrm{MN}, \mathrm{JK}, \mathrm{BN}$ performed and designed study. The multicenter CHIB 201 trial was sponsored by Novartis Pharma, Basel, Switzerland. The long term evaluation was not supported. All authors received travel grants and/or lecture fees from Novartis.

\section{References}

1. Amlot PL, Rawlings E, Fernando ON, et al. Prolonged action of a chimeric interleukin-2 receptor (CD25) monoclonal antibody used in cadaveric renal transplantation. Transplantation. 1995; 60:748-756.

2. Kahan BD, Rajagopalan PR, Hall M. Reduction of the occurrence of acute cellular rejection among renal allograft recipients treated with basiliximab, a chimeric anti-interleukin-2-receptor monoclonal antibody. United States Simulect Renal Study Group. Transplantation. 1999; 67:276-284.

3. Nashan B, Moore R, Amlot P, Schmidt AG, Abeywickrama K, Soulillou JP. Randomised trial of basiliximab versus placebo for control of acute cellular rejection in renal allograft recipients. CHIB 201 International Study Group. Lancet. 1997;350:1193-1198.

4. Vincenti F, de Andres A, Becker T, et al. Interleukin-2 receptor antagonist induction in modern immunosuppression regimens for renal transplant recipients. Transplant Int. 2006;19:446-457.

5. Matas AJ, Gilligham KJ, Payne WD, Najarian JS. The impact of an acute rejection episodes on long-term renal allograft survival (t1/2). Transplantation. 1994;57:857-859.

6. Tesi RJ, Henry ML, Elkhammas EA, Furguson RM. Predictors of long-term primary cadaveric renal transplant survival. Clin Transplant. 1993;7:345-352.

7. Furguson R. Acute rejection episodes: best predictor of long-term primary cadaveric renal transplant survival. Clin Transplant. 1994;8:328-331.

8. Hibberd AD, Trevillian JH, Wlodarzcyk JH, et al. Cancer risk associated with ATG/OKT3 in renal transplantation. Transplant Proc. 1999;31:1271-1272.

9. Swinnen LJ, Costanzo-Nordin MR, Fisher SG, et al. Increased incidence of lymphoproliferative disorder after immunosuppression with the monoclonal antibody OKT3 in cardiac-transplant recipients. $N$ Eng $J$ Med. 1990;323:1723-1728.

10. Webster AC, Playford EG, Higgins G, Chapman JR, Craig JC. Interleukin 2 receptor antagonists for renal transplant recipients: a metaanalysis of randomized trials. Transplantation. 2004;77:166-176.

11. Cockcroft DW, Gault MH. Prediction of creatinine clearance from serum creatinine. Nephron. 1976;16:31-41.

12. Wabbijn M, Balk AH, van Dormburg RT, et al. Ten-year follow-up of recipients of a kidney or heart transplant who received induction therapy with a monoclonal antibody against the interleukin-2 receptor. Exp Clin Transplant. 2004;2:201-207.

13. Beaudreuil S, Durrbach A, Noury J, et al. Long-term results (10 years) of a prospektice trial comparing Lo-tact-1 monoclonal antibody and anti-thymocyte globulin induction therpy in kidney transplantation. Transplant Int. 2006;19:814-820. 
14. Kyllönen LE, Eklund BH, Pesonen EJ, Samela KT. Single bolus antitymocyte globulin versus basiliximab induction in kidney transplantation with cyclosporine tripple immunosuppression: efficacy and safety. Transplantation. 2007;84:75-82

15. Chapman JR, Webster AC. Cancer after renal transplantation: The next challenge. Am J Transplant. 2004;4:841-842.
16. Kasiske BL, Snyder JJ, Gilbertson DT, Wang C. Cancer after kidney transplantation in the United States. Am J Transplant. 2004;4:905-913.

17. Kessler M, Jay N, Molle R, Guillemin F. Excess risk of cancer in renal transplant patients. Transplant Int. 2006;19:908-914. 